A. Alshevskaya ${ }^{3}$, A. Moskalev ${ }^{3}$, I. Kriulin ${ }^{1,2}$. ${ }^{1}$ Sechenov First Moscow State Medical University (Sechenov University), Moscow, Russian Federation; ${ }^{2}$ National Medical Research Center of Children's Health, Moscow, Russian Federation; ${ }^{3}$ Biostatistics and Clinical Trials Center, Novosibirsk, Russian Federation

Background: Early-onset form of systemic juvenile idiopathic arthritis (sJIA) often presents severe disease course. Choosing the optimal therapy option as first-line treatment is necessary for rapid improvement of patients' quality of live and prevention of further radiologic progression.

Objectives: To evaluate the long-term effectiveness and safety of tocilizumab (TOC) in SJIA patients depending on the duration of the disease treated in the National Medical Research Center of Children`s health, Moscow, Russia.

Methods: The study was conducted as a subanalysis of the prospective cohort study to evaluate the efficacy of biologics in children with SJIA. Analysis included sJIA patients younger than 4 years of age at the moment of TOC initiation. Patients were divided into 2 groups: with disease duration shorter than 6 month (ShorterDD group, $\mathrm{n}=35$ ) and more than 6 month (LongerDD group, $\mathrm{n}=19$ ). Treatment efficacy was evaluated according to the dynamics of clinical and laboratory signs using the ACRPedi criteria. The Wallace criteria were used to evaluate whether or not remission had been achieved. Treatment safety was evaluated according to the data presented in the Adverse Event Reports.

Results: TOC was first biologics in 34/35 (97.1\%) patients in ShorterDD group and $18 / 19(94.7 \%)$ patients in LongerDD group. Groups were comparable in terms of disease activity at TOC initiation with $100 \%$ of patients presented active systemic features. $31 / 35$ (88.6\%) patients in ShorterDD group and 17/19 (89.5\%) patients in LongerDD group have median 3 (IQR 1: 6) and 5 (IQR 3: 7.5) active joints, respectively $(p=0.119)$. JADAS-71 level was $17.14 \pm 6.25$ ShorterDD group and $17.36 \pm 5.45$ in LongerDD group $(p=0.895)$.

TOC showed high efficacy after first months of treatment with only $6 / 35$ (17.1\%) patients in ShorterDD group and 7/19 (36.8\%) in LongerDD group remained with active systemic features $(p=0.181)$. JADAS-71 level decreased to 0 points $26 / 35$ patients $(74.3 \%)$ in ShorterDD group and in $11 / 19$ patients $(57.9 \%)$ LongerDD group ( $p=0.237$ ). After 3 month of treatment, WID was achieved by $27 / 35$ patients $(77.1 \%)$ in ShorterDD group and by $9 / 19$ patients $(47.4 \%)$ LongerDD group ( $p=0.038$ ). ACR Pedi $50 / 70 / 90$ was achieved by $88.6 \% / 85.7 \% / 80 \%$ of patients in ShorterDD group and by $84.2 \% / 73.7 \% / 68.4 \%$ of patients in LongerDD group after 1 months of treatment and in $77.1 \% / 74.3 \% / 74.3 \%$ and $84.2 \% / 78.9 \% / 68.4 \%$, respectively, after 3 months of treatment.

Conclusion: Initiation of tocilizumab treatment in SJIA patients under 4 years of age is highly effective. However, early treatment within first 6 month after disease onset had advantages in speed of reaching an inactive disease as soon as after 3 months of therapy.

Disclosure of Interests: Elizaveta Krekhova: None declared, Ekaterina Alexeeva Grant/research support from: Roche, Pfizer, Centocor, Novartis, Speakers bureau: Roche, Novartis, Pfizer., Tatyana Dvoryakovskaya: None declared, Ksenia Isaeva: None declared, Aleksandra Chomakhidze: None declared, Evgeniya Chistyakova: None declared, Olga Lomakina: None declared, Rina Denisova: None declared, Anna Mamutova: None declared, Anna Fetisova: None declared, Marina Gautier: None declared, Dariya Vankova: None declared, Meyri Shingarova: None declared, Alina Alshevskaya: None declared, Andrey Moskalev: None declared, Ivan Kriulin: None declared

DOI: 10.1136/annrheumdis-2020-eular.5770

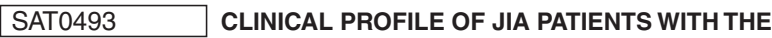 CERVICAL SPINE INVOLVEMENT: A SINGLE CENTER RETROSPECTIVE CONTINUOUS STUDY.}

$\underline{\text { R. Raupov }}^{1}$, L. Sorokina ${ }^{2,3}$, M. Dubko ${ }^{1}$, L. Snegireva ${ }^{1}$, T. Likhacheva ${ }^{1}$, A. Santimov ${ }^{1}$, E. Gaidar ${ }^{1}$, E. Isupova ${ }^{1}$, I. Chikova ${ }^{1}$, T. Kornishina ${ }^{1}$, V. Masalova ${ }^{1}$, M. Kostik ${ }^{1,3}$. 'Saint Petersburg State Pediatric Medical University, Saint Petersburg, Russian Federation; ${ }^{2}$ Leningrad Regional Children's Hospital, Saint Petersburg, Russian Federation; ${ }^{3}$ Almazov National Medical Research Centre, Saint Petersburg, Russian Federation

Background: JIA is the most common chronic condition in pediatric rheumatology. The cervical spine (CS) involvement is associated with severe disease activity and disability and has been recognized as a factor of a poor prognosis. Data about the CS involvement is contradictory due to silent CS involvement in some patients.

Objectives: the aim of our study was to provide a clinical profile of the patients with the CS involvement.

Methods: 753 patients for last 10 years with JIA were analyzed. Patients were divided depending on the CS involvement, which was confirmed by clinical (pain, LOM) and radiological features (effusion in the CS joints). We evaluated active joints and routine tests, such as CRP, ESR, ANA-positivity and HLA B27
Results: The CS involvement was in 101 patients (13.4\%). The data are in the table. The CS involvement was more frequently associated with joints of upper body, such as TMJ (23.7\% vs $2.9 \%, p=0.000001)$, shoulder $(29.7 \%$ vs $2.9 \%$ $\mathrm{p}=0.000001)$, elbow $(34.2 \%$ vs $12.2 \%, p=0.000001)$, wrist $(61.4 \%$ vs $21.8 \%$, $p=0.0000001)$, MCP (43.6\% vs $18.4 \%, p=0.0000001)$, PIP (52.5\% vs $21.3 \%$, $p=0.0000001)$, DIP $(23.8 \%$ vs $7.1 \%, p=0.0000001)$ and hip ( $44.6 \%$ vs $16.6 \%$, $\mathrm{p}=0.0000001)$, and ankle $(60.4 \%$ vs $40.2 \%, p=0.0001)$ from lower body.

\begin{tabular}{lccc}
\hline Parameters & CS, yes $(\mathrm{n}=101)$ & $\mathrm{CS}, \mathrm{no}(\mathrm{n}=652)$ & $\mathrm{p}$ \\
\hline Female, $\mathrm{n}(\%)$ & $69(68.3)$ & $388(59.5)$ & 0.092 \\
ANA-positivity, $\mathrm{n}(\%)$ & $22 / 57(38.6)$ & $190 / 403(47.2)$ & 0.226 \\
HLA B27-positivity, $\mathrm{n}(\%)$ & $12 / 33(36.4)$ & $88 / 275(32.0)$ & 0.613 \\
Onset age, years & $5.3(2.7-10.1)$ & $6.1(3.0-10.4)$ & 0.241 \\
ESR, mm/h & $12.0(5.0-31.0)$ & $7.0(3.0-18.0)$ & 0.0006 \\
CRP, mg/l & $3.9(0.0-20.0)$ & $1.1(0.0-9.2)$ & 0.002 \\
Active joints, $\mathrm{n}(\%)$ & $16.0(9.0-28.0)$ & $5.0(3.0-10.0)$ & 0.000000 \\
Time before remission, years & $2.9(1.5-5.1)$ & $2.2(1.1-4.6)$ & 0.046 \\
Oligoarthritis & $5(5.0)$ & $199(30.5)$ & 0.0000001 \\
Polyarthritis & $48(48.0)$ & $217(33.3)$ & \\
Psoriatic arthritis & $7(7.0)$ & $33(5.1)$ & \\
Enthesitis-related arthritis & $22(21.8)$ & $164(25.2)$ & \\
Systemic arthritis & $19(18.9)$ & $39(6.0)$ & \\
Uveitis, $\mathrm{n}(\%)$ & $9 / 76(11.9)$ & $107 / 444(24.1)$ & 0.018 \\
Oral glucocorticosteroids, $\mathrm{n}(\%)$ & $37(36.7)$ & $115 / 651(17.7)$ & 0.00001 \\
Biologic, $\mathrm{n}(\%)$ & $68(67.3)$ & $283(43.4)$ & 0.000007 \\
Remission, $\mathrm{n}$ (\%) & $57(56.4)$ & $428(65.6)$ & 0.072 \\
Flare, $\mathrm{n}(\%)$ & $10(9.9)$ & $128 / 651(19.7)$ & 0.018 \\
\hline
\end{tabular}

Conclusion: The main risk factors of CS involvement in JIA were polyarthicular and systemic arthritis, high inflammatory activity and involvement of joints of upper body. Patients with CS involvement required more often biologics.

Disclosure of Interests: None declared

DOI: 10.1136/annrheumdis-2020-eular.3841

\section{SAT0494 RITUXIMAB IN REFRACTORY PEDIATRIC RHEUMATIC DISEASES: FOCUS FOR THE SAFETY IN REAL CLINICAL PRACTICE}

M. Kaleda ${ }^{1}$, I. Nikishina ${ }^{1}$, E. Nikolaeva ${ }^{1}$, S. Arsenyeva ${ }^{1}{ }^{1}$ V. A. Nasonova Research Institute of Rheumatology, Pediatric Department, Moscow, Russian Federation

Background: Rituximab (RTX) is now approved only for pediatric patients (pts) 2 years of age and older with granulomatosis with polyangiitis or microscopic polyangiitis, but it has been used with success to treat another rheumatic diseases $(\mathrm{RD})$ in children despite the status "of label".

Objectives: to analyze the safety of RTX in children with various RD who did not respond to conventional therapy.

Methods: In our retrospective study safety data was analyzed for all pts, who received at least one infusion of RTX. The dose of RTX was established as $375 \mathrm{mg} / \mathrm{m}^{2}$ of body surface area, administered by intravenous infusion once weekly for 1 to 4 weeks, depending on the CD19 lymphocyte count.

Results: 81 patients with RD, who received RTX, were included: 38 (46.9\%) with systemic lupus erythemathosis (SLE), 16 (19.75\%) - JIA, polyarthritis (2 pts - RF negative, 14 - RF positive), 9 (11.1\%) - systemic JIA (sJIA), 6 (7.4\%) - systemic sclerosis (SSc), 5 (6.2\%) - primary Sjogren's syndrome (pSS), 2 $(2.5 \%)$ - juvenile dermatomyositis (JDM), 4 (4.9\%) - mixed connective tissue disease (MCTD) and 1 with livedoid vasculitis (LV). Most were female - 69 $(85.2 \%)$. The median age at onset -11.6 years [interquartile range (IQR) 7.9 14.3], median age of the starting therapy - 15.2 [IQR 12.5; 16.85] and median disease duration - 2.8 [IQR 1.0; 4.6]. 53 pts (65.4\%) reported more than one course of RTX, maximum - 10. The median time between each course was 182 days [IQR 156-315]. The RTX was effective in 95\% pts, ineffective in 5\% (2 pts with SJIA, 2 pts with SLE and macrophage activation syndrome (MAS)). Adverse events (AE) were recorded in $23(28.4 \%)$ pts, included upper respiratory tract infections $-7(8.6 \%)$, urinary tract infections $-2(2.5 \%)$, short-term infusion reactions that did not require discontinuation of therapy $-2(2.5 \%)$, clinically insignificant neutropenia (grade I-II) - 4 (4.9\%), decrease of IgG level was detected in 14 (17.5\%) pts (median $5.5 \mathrm{~g} / \mathrm{l}$ [IQR 4.0; 6.9]). The infection rate in pts with a low IgG level was $35.7 \%$, in pts with neutropenia wasn't recorded. Serious AE were recorded in $16(19.7 \%)$ pts: sepsis - 4, pneumonia - 3, herpes zoster -1 , serious infusion reactions -2 , serious postinfusion reactions within 3 to 10 days -4 ( 3 - MAS, 1 - hemorrhagic vasculitis), death -2 pts with SLE and MAS (therapy of RTX was inefficiency). In general, various AE were registered in $55.6 \%$ of pts with SJIA, in $52.6 \%$ of pts with SLE, $50 \%$ of pts with SSc and JDM, and $80 \%$ of pts with pSS. Discontinuation of therapy due to SAE was observed in 15 pts (18.5\%) 
Conclusion: Our study demonstrated that RTX is highly effective in children with RD, the majority with SLE, but the safety data obtained indicate the need for careful monitoring of therapy, primarily taking into account the frequency of infections. A decrease in IgG level was observed in a small proportion of pts and did not correlate with the incidence of infections. The frequency of serious infections was low.

Disclosure of Interests: None declared

DOI: 10.1136/annrheumdis-2020-eular.2496

\section{SAT0495 LONG TERM OUTCOME OF JUVENILE IDIOPATHIC ARTHRITIS IN ADULTHOOD; THE MONOCENTRIC EXPERIENCE OF 520 PATIENTS FOLLOWED FOR 20 YEARS IN A TRANSITION TERTIARY CLINIC OF PEDIATRIC RHEUMATOLOGY.}

1. Pontikaki ${ }^{1}$, S. Carbogno ${ }^{2}$, F. Corona ${ }^{2}$, A. Petaccia ${ }^{2}$, R. Cimaz ${ }^{1} .{ }^{1}$ ASST-PINICTO, Chair of Pediatric Rheumatology, University of Milan, MILAN, Italy; ${ }^{2}$ IRCCS Cà GRANDA Ospedale Maggiore Policlinico, MILAN, Italy

Background: Juvenile Idiopathic Arthritis (JIA) is a chronic pediatric inflammatory disease that shows many differences compared to adult-onset arthritis. The different clinical manifestations, the assessment and the management of JIA is the reason that the transition from childhood to adulthood is an important multidimensional process that emphasizes a lot of aspects.

Objectives: To describe the long-term outcome of JIA

Methods: Five-hundred and twenty patients affected by JIA and referred to a transition care rheumatology tertiary centre were considered between 1999 and 2019. The outcome assessment included remission, disease duration, medications, number of prosthesis implantation, pregnancies, mortality and social integration (employment status and educational level).

Results: A hundred and thirty-eight (26\%) males and $382(73 \%)$ females were included; $157(30 \%)$ patients were lost to follow up. The mean age of the patients was $27(18-57)$ years, with a mean age at onset of 8 years and an average disease duration of 19 years. Subtypes of JIA at disease onset included 252 (48\%) oligoarthritis, $134(26 \%)$ polyarthritis, $64(12 \%)$ systemic arthritis, $22(4 \%)$ psoriatic arthritis, $43(8 \%)$ enthesitis related arthritis and 1 $(0.1 \%)$ undifferentiated arthritis. Ninty-three $(18 \%)$ patients suffered of uveitis. Ninty-five implant prosthesis and 16 arthrodesis were recorded. At follow up $198(38 \%)$ patients were on remission of which $107(20 \%)$ off medication. Among the 322 patients still on medication, 84 (16\%) were under treatment with oral steroids, 226 (43\%) with sDMARDs and 249 (40\%) with bDMARDs. Five deaths (1\%) occurred in this cohort. Two hundred and thirty-five subjects had a higher educational level, 327 had an employment. We have data of twenty-nine pregnancies. The transition age was considered after the age of 16 years old. The key word for the management of this cohort was the multidisciplinary approach towards each patient, with the collaboration of other specialists (ophthalmologist, orthopedic, dermatologist, gastroenterologist, obstetric and psychologist).

Conclusion: In the era of biologic therapy the long-term outcome of JIA underwent an outstanding improvement regarding a lot of variables. Two hundred and thirty-two patients were still followed, not only because of the continuation of the biological therapy, but also for a multidisciplinary care even during remission. JIA often persists over the adulthood, therefore the long term follow-up and care of these patients needs to be conducted by a rheumatologist expertized in JIA in collaboration with other specialists.

Disclosure of Interests: None declared

DOI: 10.1136/annrheumdis-2020-eular.5540

\section{SATURDAY, 06 JUNE 2020}

\section{Basic and translational science in paediatric rheumatology}

\section{SAT0496 \\ INTERROGATING THE CIRCULATORY IMMUNE ARCHITECTURE OF ENTHESITIS-RELATED ARTHRITIS}

S. H. Tay ${ }^{1,2}$, J. Y. Leong' ${ }^{2}$, M. Wasser ${ }^{2}$, A. J. M. Lim², P. Chen², J. G. Yeo ${ }^{1,2,3}$, T. Arkachaisri ${ }^{1,4}$, S. Albani ${ }^{1,2,3}{ }^{1}$ Duke-NUS Medical School, Singapore, Singapore; ${ }^{2}$ Translational Immunology Institute, SingHealth/Duke-NUS Academic Medical Centre, Singapore, Singapore; ${ }^{3} K K$ Women's and Children's Hospital, Singapore, Singapore; ${ }^{4} K K$ Women's and Children's Hospital, Rheumatology \& Immunology Service, Singapore, Singapore
Background: Enthesitis-related arthritis (ERA) is one of the most common subtype of juvenile idiopathic arthritis (JIA) in Asia ${ }^{1}$. It carries a poor prognosis, but limited knowledge of its pathogenesis hampers clinical diagnosis and treatment. Objectives: We hypothesise multiple aberrations from the healthy immunome culminating in an imbalance between the immune effector and regulatory cel subsets as key for driving ERA pathogenesis. Thus, we employed a comprehensive high-dimensional interrogative strategy using mass cytometry to assess the ERA immune architecture ${ }^{2}$

Methods: We examined peripheral blood mononuclear cells from 30 ERA patients (15 with active sacroilitis, 15 without active sacroilitis) within the first two years of disease and 30 healthy paediatric controls with mass cytometry, using two extensive antibody panels encompassing key lineage and functiona markers. Dimensional reduction and unsupervised clustering were performed to identify immune cell subsets differentially present in ERA patients. Manual gating was performed to further describe observed differences in subset frequencies. These subsets were statistically evaluated with reference to the healthy cohort and their association with disease activity determined.

Results: We identified broad differences in the ERA circulatory immune architecture that involved both innate and adaptive immune cell populations, notably with the enrichment of naive CD4+ T cells as well as depletion of cytolytic NK cells (CD56 ${ }^{\text {dim }}$ CD16+). The chemotactic profiles of their subsets also differed in ERA patients, which underscores their migratory capacity and hence potential effector role in the ERA arthritic microenvironment. In addition, there were some dissimilarities in the circulatory immunome of ERA patients with active sacroiliitis as compared to those without, which alludes to a possible mechanistic basis behind the disease complication.

Conclusion: This is the first study, via deep parameterisation afforded by mass cytometry, to demonstrate a concomitant dysregulation of both innate and adaptive immune cell subsets in ERA patients. Further mechanistic studies of these immune cell subsets and their functional networks will inform the development of diagnostic and prognostic markers that can reliably predict clinical fate in ERA, thereby complementing clinical assessment in the care of ERA patients.

References:

[1] Arkachaisri et al. Paediatric rheumatology clinic in Southeast Asia: are we different? Rheumatology (Oxford). 2017.

[2] Tay et al. Immunomics in pediatric rheumatic diseases. Front Med (Laus anne). 2019.

Disclosure of Interests: None declared

DOI: 10.1136/annrheumdis-2020-eular.5125

\section{SAT0497 \\ A PILOT PROTEOMIC ANALYSIS OF PLASMA BIOMARKERS IN IGA VASCULITIS}

S. Demir ${ }^{1}$, M. Sardan ${ }^{2}$, I. Yet ${ }^{3}$, E. Sag ${ }^{1}$, Y. Bilginer ${ }^{1}$, Ö. Celikbıcak ${ }^{4}$, S. Özen ${ }^{1}$. ${ }^{1}$ Hacettepe University Faculty of Medicine, Department of Pediatric Rheumatology, Ankara, Turkey; ${ }^{2}$ Hacettepe University Faculty of Medicine, Hunitek Research Center, Ankara, Turkey; ${ }^{3}$ Hacettepe University Faculty of Medicine, Department of Bioinformatics, Ankara, Turkey; ${ }^{4}$ Hacettepe University Faculty of Medicine, Department of Chemistry, Ankara, Turkey

Background: IgA vasculitis/ Henoch Schönlein Purpura (IgAV/HSP) is the most common vasculitis of childhood, characterized by the $\lg \mathrm{A} 1$ immune deposits in the small vessels. Although it is very common, the understanding of its molecular pathogenesis is still very limited.

Objectives: We aimed to analyse plasma proteomes of IgAV/HSP patients using nano liquid chromatography - tandem mass spectrometry (nLC-MS/MS) to investigate the disease pathogenesis.

Methods: IgAV/HSP was diagnosed according to the Ankara criteria in 2008 (1). Five active IgAV/HSP patients and two age and gender-matched health controls were enrolled in this pilot study. Serum samples from subjects were collected on the same day of IgAV/HSP diagnosis and before steroid or other immunosuppressive treatment initiated. Sample preparation was carried ou using PreOmics iST Kit. We investigated the alteration of serum proteome using the nano LC-MS/MS approach. Bruker raw files were analyzed using the proteomics software Max Quant (1.6.7.0). The human reference proteome set from UniProt was used to identify proteins. Proteomic data were analyzed with Perseus 1.6.7.0.

Results: The data file includes peptide and protein identification, accession numbers, protein and gene names, sequence coverage and label free quantification (LFQ) values of each sample. 345 proteins were reported per sample. Identifications from the reverse decoy database, identified by site only and known contaminants were excluded. Data were log transformed. Two sample T-test was performed between groups. We identified 23 significantly different expressed proteins (Table 1). Mainly the differentially expressed proteins were in the $\mathrm{Ig}$ and complement pathway, innate immune inflammatory, 